\title{
A Straightforward Design and Implementation of Real Time Pressure Monitoring System in Water Pipe
}

\author{
Dwi Astharini*, Arizka \\ Program Studi Teknik Elektro, Fakultas Sains dan Teknologi, \\ Universitas Al Azhar Indonesia, Jl. Sisingamangaraja, Jakarta 12110 \\ *Penulis untuk Korespondensi: astharini@uai.ac.id
}

\begin{abstract}
This paper reports the development of a realtime system for pressure monitoring inside water pipe. In water supply industry it is valuable to know the water pressure inside the pipe. Thus the system created consisting hardware parts in compact form along with software in laptop for high mobility. In the hardware part, pressure gauge is chosen as sensor, and a conversion of the data into frequency is decided for better communication with the computer. The process in the software includes data captures, conversion, and display using visual basic MSviewer.
\end{abstract}

Keywords - Real Time, Pressure monitoring, Frequency conversion

\section{INTRODUCTION}

$\mathrm{O}$ ne key in increasing productivity is monitoring system, by which optimal performance can be achieved with low maintenance and especially reparation costs. The device system described is used to know the amount of pressure in a pipe for sampled time. From these measurements we obtain the water pressure data, monitoring them to overcome any malfunction to avoid large damage of the system. The data is also kept for analysis, such as user behaviour, or for the need of modification.

The resulting applications can be used to monitor the water usage in general, and further analysis such as the pattern on the water consumption, abnormal behaviour and others.

The paper is divided into several parts. Second part of this paper will discuss some recent developments on the subject. Third part is methodology and system design. The fourth consist of the hardware implementation, and the data handling before presenting the conclusion on the fifth part.

\section{LITERATURE REVIEW}

The system in [1] is called Application for Water Leak Detection (AIDA), basically a set of actuators installed in the hydraulic system, and sensor device to detect leaks. Readings from sensors are wirelessly processed by a microcontroller coorporating warning via SMS to system user. The main objective here is to detect and handle water leakage in a short time.

The paper [2] presents PipeSense, an integrated inpipe water monitoring system aiming to monitor water contaminations and leakages along distribution pipes, employing near field RFID WSNs.

Paper [3] used negative $\mathrm{P}$ wave method and flow balance method to detect and locate leakage location.

\section{METHODOLOGY AND SYSTEM DESIGN}

The tool designed is used to determine the water pressurewithin the pipes by punching a small hole (or a few) in the side of the pipe. Valve will be installed in the hole as connect or to the sensor, water is to enter the hose connected to the pressure sensor and the pressure will be projected in a circuit. The whole circuit used will change information signal from mechanical to an audio frequency, which is then send into aPC/laptop using an audio cable. Here pressure inside the pipe is to be monitored by using visual basic program. The monitoring system can show the amount ofwater pressure in each second. 
The instrument design is shown in Figure 1. The scenario starts from the inputs in the form of pressure that occurs in the pipeline that will drive the membrane in the gauge pressure sensors. Pressure sensor will transform it into voltage outputs and then this electrical signal is later send to be converted into sinusoids with the pressure information coded in its frequency. This form of coding is chosen as way of analog to digital conversion, in the same time simplify the data transfer to PC, so that the system will utilize the audio line in.

The gauge pressure sensor used is MPXM2010GSh with four legs: two for input voltage $V^{+}$dan $V^{-}$, and two other for the outputs. The two output legs show the voltage difference which is interpreted as a measure of pressure in the pipe. But the difference is very small with voltage range between $0 \mathrm{mV}-25 \mathrm{mV}$ for the chosen component. It is therefore necessary to amplify the voltage by using Op Amp in order to make signal larger for further process.

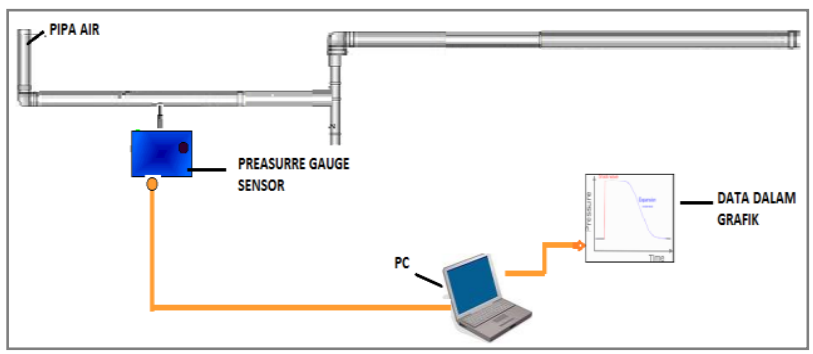

Figure 1.System Design

\section{IMPLEMENTATION RESULT}

\section{IV.1 Design Implementation and Component Configuration}

The electronic parts of the system design is implementad as the schematic shown in Figure 2. Pressure sensor is used to measure pressure within the pipe stem. The sensor works by utilizing the movement of water pressure inside the pipe [4]. Measurement is done using a probe that is injected to the main pipe line network. IC pressure sensor using a type MPXM2010GS with pressure gauge is utilized, which is a silicon photo resistive pressure sensors providing highly accurate and linear voltage output, directly proportional to the applied pressure. The sensor is a single monolithic silicon diaphragm with a strain gauge and thin film resistor network integrated on-chip. The chip is laser trimmed to accommodate precise span, offset calibration, and temperature compensation. Operating pressure range measurable is of 0 to 10 $\mathrm{kPa}$ with pressure axial port type, having the sensitivity $2.5 \mathrm{mV} / \mathrm{kPa}$. This component operates with $6 \mathrm{~mA}$ supply current and supply voltage of 10$16 \mathrm{~V}$, in operating temperature range of 40 to $125^{\circ} \mathrm{C}$.

The Op Amps used are of IC MC33179P type. They are used in receiving a voltage from the pressure transducer which is to be amplified and turned into a differential voltage

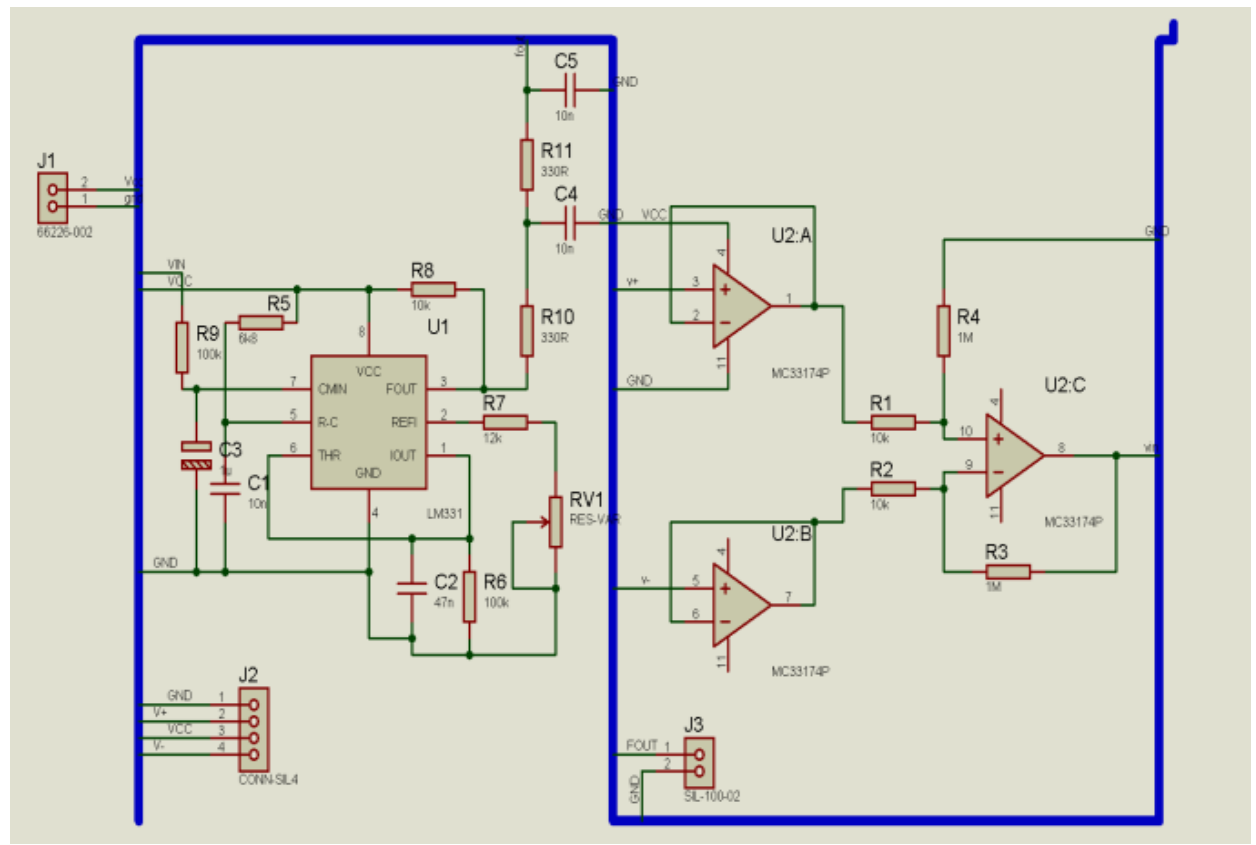

Figure 2. Schematic of Overall Equipment 
The differential voltage resulted then went into the LM331. This device works to convert the voltage to frequency as the analog to digital conversion. The frequency output of this device will be the output on this electronics subsystem. The information coded in signal frequency out of this device is digital information. In this part it is necessary to use Low Pass Filter to maintain the signal from several noises, which is already available in the use of Op Amp MC33179P.

\section{IV.2 System Testing}

Originally the communication was setup using VB 6.0. But soon it was realized that it does not support the communication between RC Plug and soundcard nor make it simple to send data to the system. So the .NET Framework under C\#.NET is then used, which supports better for this effort. Communication between hardware and software is better on this framework because the .NET itself provides rich function and components around these parts. It is also supported by more Operating Systems than the successor, giving more freedom in its mobility of usage.

Figure 3 is showing the testbed for the real time commication between hardware and the laptop.

The pressure in pipe release force to the IC then the Audio card read from its RC plug port. Then the Audio sound card gives away the signal into 2 channels. The system in development utilize both channels of right and left, and send them for data process. System translates the value from soundcard (byte to double) then shown as the graphic, as the capture shown in Figure 4.

The data above is to be written on database, therefore the _wave Left and _wave Right representing each channel were at first processed and analysed. And as in the Figure 4, both waves are depicted on monitoring display. This display is also used to monitor the communication line states, to see whether the connection is as it should be.

On the real time monitoring display are four buttons for essential functions.

On click upon the show report, the system calls report of pressure to show the frequency graphic, as shown in figure 5 .

This frequency graphic is still showing two sets of data from the audio channel pair. One of them seemed to be an LPF, passing only thresholded frequency. This lead to decision in using sets of data from the other audio channel.

Once it is decided which channel is to be taken, the pressure data can directly be obtained from the frequency. An example of the graphic form of it is shown in Figure 6. From the picture we can see high and low pressure.

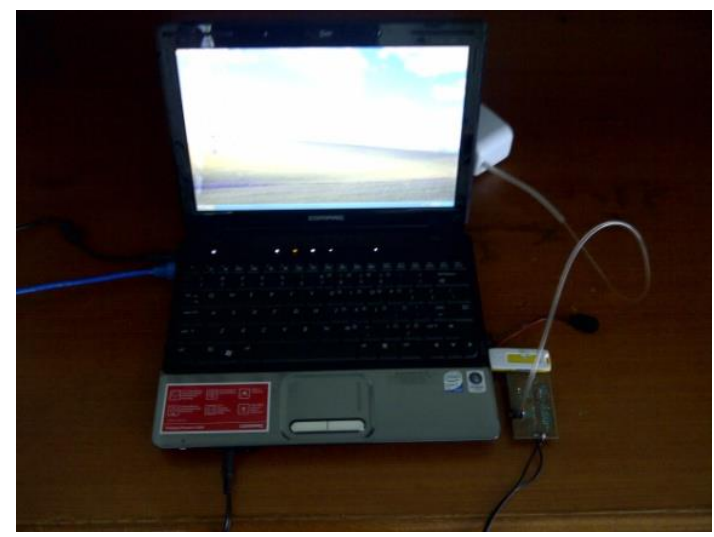

Figure 3. Real Time Communication Testbed

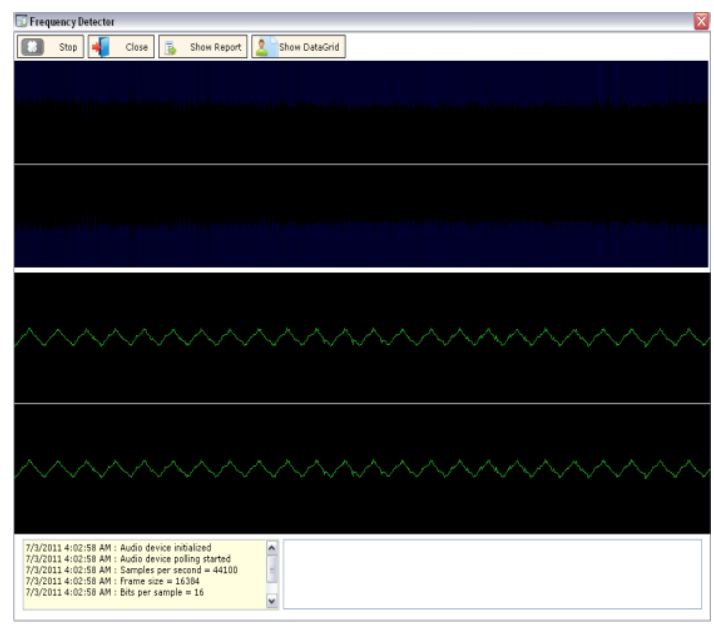

Figure 4. Monitoring display of frequency coded data

\section{CONCLUSION}

The tool developed can identify water pressure condition in pipeby using pressure sensors. The sensor utilized is a mechanical sensor that will push the membrane and transducer. The electrical signal produced from the transducer is treated in a few steps to change the information in form of frequency data, which is fed to PC through audio inputports. The software part fetch the data as audio, then read its frequency for every second. 
This information is then converted to pressure data in real time, to be displayed, stored, and use for further analysis.

\section{REFERENCES}

[1] Gama-Moreno L., Reyes J., Sánchez M., OchoaFranco C., Noguerón C., "Instrumentation of a water-leaks detection system controlled via the Short Message Service through the GSM Network", Proceedings of 2010 Electronics, Robotics and Automotive Mechanics Conference

[2] Nasir A., Soong B., "PipeSense: A framework architecture for in-pipe water monitoring system", Proceedings of the 2009 IEEE 9th Malaysia International Conference on Communications
[3] Xiao-Li Ca, Chao-Yuan Jiang, Si-Yuan Gan, "Leakage Monitoring and Locating Method of Water Supply Pipe Network", Proceedings of the Seventh International Conference on Machine Learning and Cybernetics, 2008

[4] Fraden, Jacob. 2004 "Handbook of Modern Sensors: Physics, Designs and Application". Springer. New York.

[5] Anon. "Sensor Evolution", http://www. sensorsmag.com/sensors-mag/sensor-evolution7245, accessed: June 42011

[6] Anon. http://www.alldatasheet.com/datasheetpdf/pdf/93836/MOTOROLA/MPXM2010GS.html, Accessed June 82011

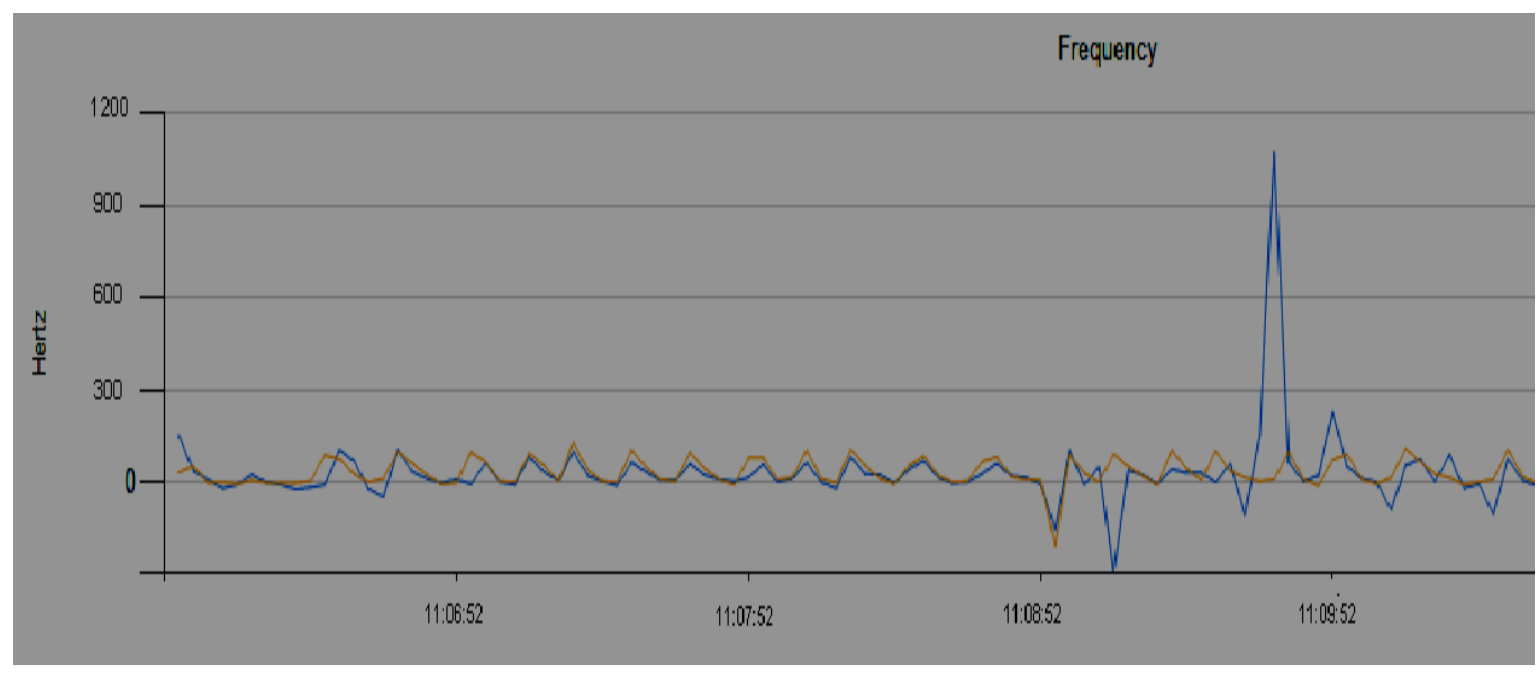

Figure 5. Frequency Graphic

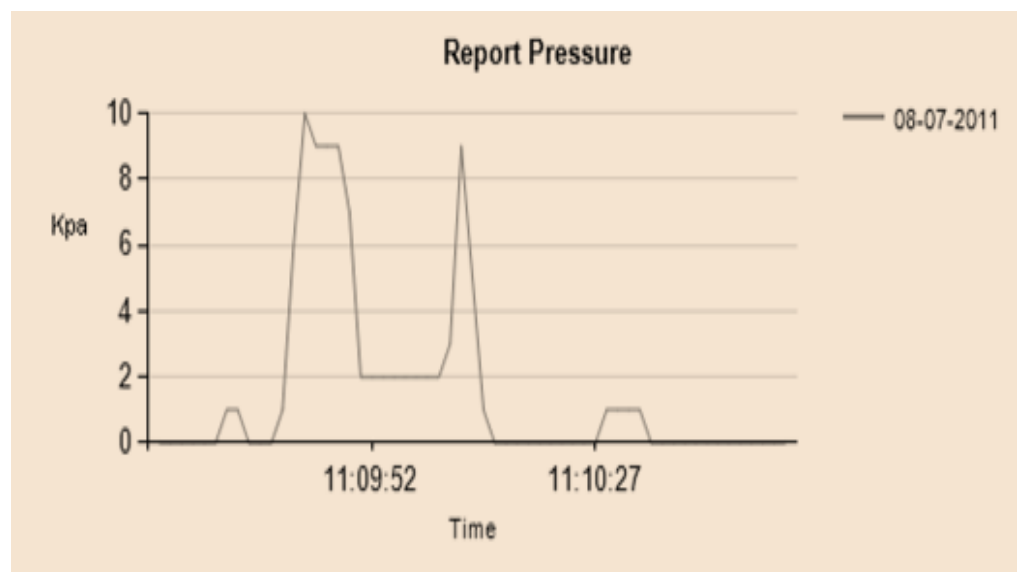

Figure 6. Pressure Monitor 